\author{
Andriej Kotin \\ ORCID: 0000-0003-1742-2421 \\ Universität Zielona Góra, Zielona Góra
}

DOI: $10.19195 / 0435-5865.143 .2$

\title{
Dies- und jenseits des Flusses - Die Struktur als sinntragendes Element von Ludwig Tiecks Die Elfen
}

\begin{abstract}
s
Der vorliegende Artikel befasst sich mit der Analyse der Struktur von Ludwig Tiecks Kunstmärchen Die Elfen. Struktur wird hier nicht nur als bloße Raumkonfiguration, sondern als handlungsorientierendes und sinntragendes Textelement verstanden. Dabei wird mit einigen früheren Interpretationsversuchen polemisiert, bei denen vor allem psychologische bzw. phantastische und realistische Aspekte des Märchens im Zentrum der Untersuchung stehen. Ein extra Kapitel ist der gattungstypologischen Frage nach dem eigentlichen Genre von Die Elfen gewidmet.
\end{abstract}

Schlüsselwörter: Tieck, Märchen, Struktur, Raum, Komposition

\section{This and other side of the river - the structure as a semantic element of Ludwig Tieck's Die Elfen}

The article is based upon the author's structural analysis of Ludwig Tieck's fairy-tale Die Elfen. One should bear in mind, however, that the structure of the tale represents more than a mere spatial configuration, namely that it is a significant and intrinsically meaningful element of the entire text, integral in advancing the narrative. A polemic against previous interpretations (particularly those based on the psychological, fantastic or realistic aspects of the story) is also included. An ancillary chapter delves in the genre-specific question: should Die Elfen be considered as a fairy tale in the original sense of the word, or is the work better defined as a short novel (or "novella") which includes magical features?

Keywords: Tieck, fairy-tale, structure, space, composition

Andriej Kotin, Uniwersytet Zielonogórski, Instytut Filologii Germańskiej, Al. Wojska Polskiego 71a, 65-762 Zielona Góra, Polen, E-Mail: andriejkotin@gmail.com 


\section{Die Falle des Offensichtlichen - Zur Einleitung}

1811 entstanden und 1812 in der Phantasus-Sammlung publiziert, werden Ludwig Tiecks Die Elfen, die der Schweizer Literaturhistoriker Albert Béguin zu den besten phantastischen Texten von Tieck zählt (vgl. Béguin 2011: 274), eher selten zum Objekt literaturwissenschaftlicher Analyse. Ein zweifelloser Grund dafür ist die unübertroffene, stets aktuelle Popularität des als ,zentrales Archiv der romantischen Literatur" (Kremer 2005: 53) angesehenen Gruselmärchens Der Blonde Eckbert, dessen zahlreiche Rezeptions-, Interpretations- und Überinterpretationsversuche Achim Hölter in seinem 2005 erschienenen Artikel Über Weichen geschickt und im Kreis gejagt zusammengefasst, eingehend kommentiert und auf eine äußerst originelle Weise dem leserkreierenden Potenzial des Textes zugeschrieben hat (vgl. Höller 2005: 69-91). Dieser vielseitige Widerhall führte dazu, dass alle anderen Märchenerzählungen von Tieck entweder im Schatten Eckberts bleiben oder ausgerechnet in dessen Licht betrachtet werden. So schreibt z.B. Manfred Frank, Die Elfen seien ,unter Tiecks Märchen dasjenige, das sich am engsten an die überkommene Märchentradition anlehnt“ und somit sein „zugänglichste[s] und kinderfreundlichste[s]" (Frank, in: Meißner 2007: 347) Märchen. Bedenkt man, dass es sich dabei um einen Text handelt, in dem alle Sympathie erweckenden Gestalten am Ende sterben, während der anfangs so lieblich dargestellte Raum schließlich in absolute Verwesung gerät, so klingt ein solch optimistisches Urteil etwas voreilig. Auch Thomas Meißner unterstreicht das Ländlich-Idyllische an Tiecks Märchennovelle, indem er sie mit zwei anderen, in derselben Zeit verfassten und veröffentlichten Texten vergleicht: „Im Gegensatz zu den „Stadtmärchen“ Liebeszauber und Pokal spielen Die Elfen auf dem Land, in einer vergangenen, wenngleich unbestimmt bleibenden Zeit" (ebd.). Die überwiegend komparatistische Vorgehensweise bezieht sich allerdings nicht nur auf die Elfen, sondern auch auf das gesamte Märchenwerk von Tieck. Selbst Detlef Kremer, der die Phantasus-Märchen zwar als ,außerordentlich voraussetzungsreich und vielschichtig“ (Kremer 2005: 53) bezeichnet, macht jedoch gleichzeitig folgende fragwürdige Behauptung: „Verglichen mit der allegorischen Überkomplexität von Goethes Märchen oder der geschichtsphilosophischen und mythischen Vertracktheit von Novalis' Klingsohr-Märchen sind Tiecks Märchen tatsächlich überschaubar komponiert" (ebd.). Erstaunlich ist hier vor allem das Tertium comparationis oder, genauer gesagt, dessen Fehlen. Goethes allegorischer Überfluss sowie Novalis' philosophische bzw. mythische Anspielungen werden mit einer - Tiecks Märchen zugeschriebenen - überschaubaren Komposition verglichen. Ein überschaubar komponierter Text kann aber durchaus viele „vertrackte“ Symbole oder mehrere Allegorien enthalten, was gerade bei Tieck oft der Fall ist (als Beweis dafür könnte hier Edward Lüers 262 Seiten starke Untersuchung dienen, die gänzlich der an Jakob Böhmes theosophisches System angelehnten symbolischen Entschlüsselung von Tiecks Der Runenberg gewidmet ist (Vgl. Lüer 1997)). 
Andererseits ist z.B. Novalis' von kulturellen, geschichtlichen und philosophischen Hinweisen wimmelndes Märchen Hyazinth und Rosenblütchen - strukturell gesehen - zumindest genauso „überschaubar“ wie die Märchen von Tieck. Kurzum: Die Komposition bzw. Struktur eines literarischen Werkes gehört dem Wie, seine philosophisch-metaphorische Komplexität dagegen dem Was des Erzählten, weshalb diese zwei Ebenen - ästhetisch wie ontologisch aufs Engste miteinander verbunden bei einer narratologischen Analyse weder vermischt noch gegenübergestellt werden sollten. Bemerkenswert ist übrigens, wie einheitlich und einig sich alle in Bezug auf die in Elfen bestehenden Stereotypen sind. Resümiert man das Meiste unter dem Wenigen, was über dieses Märchen geschrieben wird (Vgl. Kováts: 452, Zugriff am 20.06.2017), so lassen sich vor allem drei Aspekte hervorheben:

1. Eine typisch märchenhafte, im Vergleich zu Der blonde Eckbert oder Der Runenberg friedliche, ja heitere Stimmung.

2. Eine typisch märchenhafte, beinahe konfliktlose, wenn auch etwas melancholisch gefärbte Handlung.

3. Eine typisch märchenhafte, unkomplizierte Struktur (Komposition), wo die Räumlichkeit eher als Setting erscheint und keine semantisch aufgeladene performative Rolle spielt.

Der vorliegende Artikel stellt den Versuch dar, Ludwig Tiecks Elfen vom vorherrschend komparatistisch orientierten Interpretationsschema loszulösen und die erwähnten stereotypen Feststellungen mittels einer größtenteils textimmanenten, möglichst expliziten Strukturanalyse in Frage zu stellen. Der Begriff „Struktur“ wird dabei im weitgefassten narratologischen Kontext einer semantisch aufgeladenen Konstruktion erzählter Wirklichkeit verstanden. In diesem Sinne ist der strukturelle Aufbau des Textes mit Jurij Lotmans Raumkonzept eng verbunden, denn die ,allerallgemeinsten sozialen, religiösen, politischen, ethischen Modelle der Welt, mit deren Hilfe der Mensch [...] den Sinn des ihn umgebenden Leben deutet, sind stets mit räumlichen Charakteristiken ausgestattet" (Lotman 1993: 313). Das Hauptanliegen der Textuntersuchung geht über die reine formale Analyse hinaus und richtet sich auf ein tieferes Verständnis derjenigen romantischen Sehnsucht, welche die ,metaphysische Obdachlosigkeit“ (Safranski 2015: 94) beider weiblichen Hauptfiguren - Marie und Elfriede - bedingt und begleitet.

\section{Die Elfen zwischen Märchen und Novelle - Gattungsspezifische Besonderheiten}

Das klassifikatorische Problem (das einen Literaturwissenschaftler eigentlich viel weniger interessieren sollte als einen Buchhändler) lässt sich im Fall von den Elfen auf die einfache Frage beschränken: Ist Tiecks Text der Märchengattung zuzuordnen oder stellt er eher eine phantastische Erzählung bzw. Novelle dar? Die Antwort 
auf diese Frage ist - aus analytischer Perspektive - nur unter der Bedingung signifikant, dass gattungsspezifische Charakteristika einen bedeutsamen Einfluss auf die Form bzw. den Inhalt eines literarischen Textes haben. Diesen Einfluss sollte man indessen weder verabsolutieren noch geringschätzen, sondern adäquat konkretisieren, und zwar als keinen unmittelbaren, wohl aber einen indirekten Einfluss. Es sind schließlich nicht die Gattungsgesetze, die den Aufbau eines künstlerischen Werkes bestimmen. Im Gegenteil: Das originelle, zum Prototyp jeweiliger Gattung gewordene Werk schafft erstmal gewisse homogene ,Spielregeln', an die sich seine späteren Nachläufer entweder halten oder sie zu modifizieren bzw. gar zu brechen suchen. Spricht man von einem klassischen „Rätsel-Lösungs-Schema der Detektivgeschichte" (Borais 2011: 104), so geht man davon aus, dass es ein bestimmtes, Vorbild“ bzw. Muster solcher Geschichten gibt, einen ursprünglichen „Archetyp“ (ebd.) (sei es E.A. Poes Der Doppelmord in der Rue Morgue oder die Scherlock-Holmes-Abenteuer von A.C. Doyle), welcher, einmal verwendet, im Nachhinein mehrmals wiederholt (in der sog. ,Trivialliteratur"), erweitert oder auch (in der sog. ,experimentellen Literatur") bewusst verstört bzw. verneint wird. In dieser Hinsicht ist die Situation mit der Märchengattung insofern kompliziert, als es keinen derartigen einzelnen Archetyp gibt, ja überhaupt geben kann, denn die ersten Gattungsvertreter, die Volksmärchen, sind sowohl autorenlos als auch zeitgeschichtlich nicht immer fixiert, so dass die Gattung an sich archetypisch bzw. mythenhaft konzipiert wird. Dennoch existieren natürlich mehrere als märchentypisch geltende Faktoren. Prüft man Die Elfen nach der An- bzw. Abwesenheit dieser Faktoren, so kann das Ergebnis schematisch folgendermaßen zusammengefasst werden:

\begin{tabular}{|l|l|}
\hline \multicolumn{1}{|c|}{ Gattungstypisch } & \multicolumn{1}{c|}{ Gattungsabweichend } \\
\hline Dorf und Wald als Handlungsräume & $\begin{array}{l}\text { abrupter Textanfang (,Wo ist denn Marie, } \\
\text { unser Kind?“ (Tieck 1983: 7)) }\end{array}$ \\
\hline $\begin{array}{l}\text { Koexistenz vom realen und phantastischen } \\
\text { Raum (die Elfen- und die Menschenwelt) }\end{array}$ & $\begin{array}{l}\text { novellenartiger Erzählstil: Wiederholungen } \\
\text { treten selten, längere und kunstvolle } \\
\text { Beschreibungen dagegen häufig auf }\end{array}$ \\
\hline $\begin{array}{l}\text { Grenzgängerfigur (ein kleines Mädchen findet } \\
\text { sich plötzlich im zauberhaften Elfenreich) }\end{array}$ & $\begin{array}{l}\text { trotz des ländlichen Handlungsraums steht } \\
\text { im Erzählzentrum eine wohlhabende, } \\
\text { kleinbürgerliche Familie }\end{array}$ \\
\hline $\begin{array}{l}\text { fabelhafte Wesen und magische Gegenstände } \\
\text { (Elfen, Zwerge, der Vogel Phönix, der Ring) }\end{array}$ & $\begin{array}{l}\text { knappe, aber exakte psychologische Portraits } \\
\text { mit Entwicklungspotenzial }\end{array}$ \\
\hline $\begin{array}{l}\text { aufgehobene Naturgesetze (Marie verbringt } \\
\text { bei den Elfen kaum einen Tag, währenddessen } \\
\text { in der Menschenwelt sieben Jahre vergangen } \\
\text { sind (ebd.)) }\end{array}$ & $\begin{array}{l}\text { keine Gut-Böse-Teilung, sowohl in Bezug } \\
\text { auf die Figuren als auch auf ihre Taten, so } \\
\text { dass weder das Gute belohnt noch das Böse } \\
\text { bestraft wird }\end{array}$ \\
\hline $\begin{array}{l}\text { das gegebene und gebrochene Versprechen } \\
\text { (niemandem die Wahrheit über die Existenz } \\
\text { des Elfenreiches zu erzählen) (ebd.) }\end{array}$ & trauriger, ja tragischer Ausklang \\
\hline
\end{tabular}


Aus der skizzierten Tabelle wird sichtbar, dass die Märchenelemente und die Züge eines realistischen Prosatextes sich in den Elfen gegenseitig ausgleichen und ergänzen. Um die verborgene, unauffällige und doch sinnstiftende Besonderheit dieser seltsamen Geschichte zu erkennen, sollte man daher ihrem Aufbau mehr Aufmerksamkeit schenken als dem, was auf der bloßen Handlungsebene passiert. In seinem Aufsatz Shakespeares Behandlung des Wunderbaren schreibt Ludwig Tieck: „Man glaubt dem epischen Dichter gleichsam auf sein Wort, wenn er nur einige Kunst anwendet, seine wunderbare Welt wahrscheinlich zu machen [...]" (Tieck 1985: 377). Nehmen wir nun diese „einige Kunst“ unter die Lupe, um zu sehen, wie eng hier das Strukturbildende mit dem Sinntragenden zusammenarbeitet.

\section{Räume und Grenzen - Der Aufbau der erzählten Welt(en)}

Die Frage nach der Struktur von Tiecks Märchennovelle ist gar nicht so einfach, wie es auf den ersten Blick scheinen mag. Angesichts des allgemeinen Einverständnisses (besser: der geltenden Opinio communis), Die Elfen seien ein generell liebenswürdiger, für Kinder durchaus geeigneter Text, sind einige amüsante Unstimmigkeiten umso bezeichnender. So stellt z.B. Detlef Kremer im oben erwähnten Statement die überschaubare Komposition von Elfen der metaphorischen Hochkomplexität von Goethes Märchen gegenüber, während Ingrid Kreuzer diese Meinung zwar teilweise bestätigt - indem sie Tiecks Text auch als unkompliziert bezeichnet -, dabei jedoch hinzufügt, diese Simplizität erinnere an Goethes Märchen (Vgl. Kreuzer 1983: 117). Dies ist nicht nur ein weiteres Beispiel für die unabwendbare Subjektivität diverser literaturwissenschaftlicher Feststellungen, sondern auch ein Beweis dafür, wie verzweigt und strittig der Strukturbegriff an sich sein kann: Es ist natürlich möglich, dass Kremer und Kreuzer den Grad an Komplexität in Goethes Märchen so divergent einschätzen, höchstwahrscheinlich werden hier aber unter derselben Bezeichnung - „Struktur“ - zwei verschiedene Textkomponenten verstanden.

Die Schwierigkeiten mit der Elfen-Struktur lassen sich recht anschaulich anhand des folgenden Zitats aus Judit Kováts’ Artikel Grenzübergänge des Wirklichen in Ludwig Tiecks Märchen „Die Elfen“ beobachten:

Die Landschaft ist [...] schon zu Beginn der Geschichte durch sichtbare Grenzen in voneinander separierte Gegenden geteilt. Das Haus der Familie liegt auf einer kleinen Anhöhe, der Fruchtgarten ist von einer Stakete umgeben, das Dorf zog sich etwas tiefer hinunter. Von dieser paradiesischen Landschaft ist ein dürrer und abgelegener Fleck, der "traurige“ und "schwarze“ Tannengrund durch einen Fluss - mal als Bach, mal als Strom genannt - getrennt. (Kováts: 454, Zugriff am 20.06.2017)

Die erwähnten Grenzen sind in der Tat ziemlich sichtbar, anscheinend aber nicht so sichtbar, wie die Autorin behauptet, denn die eigentliche Raumteilung bei Tieck unterscheidet sich von der oben dargestellten in einer kleinen, doch 
nicht unbedeutenden Nuance. Indem Kováts den Bach bzw. Strom mit dem Fluss gleichsetzt, vermischt sie Räume bzw. Teilräume, die im Textgerüst der Elfen klar voneinander getrennt werden: Den ehemaligen und den aktuellen Wohnort von Maries Eltern, Martin und Brigitte. Heißt es doch im Text (hier ist ein längeres Zitat hinsichtlich mehrerer signifikanter Einzelheiten unerlässlich):

Ihr Haus lag auf einer kleinen, grünen Anhöhe, von einem zierlichen Stakete umgeben, welches auch ihren Frucht- und Blumengarten umschloß [...]. Martin hatte von der Herrschaft das große Gut gepachtet und lebte mit seiner Frau und seinem einzigen Kinde vergnügt, denn er legte jährlich zurück und hatte die Aussicht, durch Tätigkeit ein vermögender Mann zu werden, da der Boden ergiebig war und der Graf ihn nicht drückte.

Indem er mit seiner Frau nach seinen Feldern ging, schaute er fröhlich um sich und sagte: „Wie ist doch die Gegend hier so ganz anders, Brigitte, als diejenige, in der wir sonst wohnten. Hier ist es so grün, das ganze Dorf prangt von dichtgedrängten Obstbäumen, der Boden ist voller Kräuter und Blumen, alle Häuser sind munter und reinlich, die Einwohner wohlhabend [...]. „Sowie man nur“, sagte Brigitte, „dort jenseits des Flusses ist, so befindet man sich wie auf einer andern Erde, alles so traurig und dürr; jeder Reisende behauptet aber auch, daß unser Dorf weit und breit das schönste sei.“

„Bis auf jenen Tannengrund“, erwiderte der Mann; ,schau einmal dorthin zurück, wie schwarz und traurig der abgelegene Fleck in der ganzen heitern Umgebung liegt; hinter den dunkeln Tannenbäumen die rauchige Hütte, die verfallenen Ställe, der schwermütig vorüberfließende Bach.“ (Tieck 1983: 7-8)

Somit wird die Erzählwelt in drei Handlungssektoren gegliedert: Zwei übergeordnete Haupträume - dies- und jenseits des Flusses - sowie einen untergeordneten Teilraum (Tannengrund). Ganz nach Lotmans Raumtheorie teilt die Grenze bei Tieck „den Raum in zwei disjunkte Teilräume“ (Lotman 1993: 327), wobei die Kerneigenschaft dieser Grenze in deren „Unüberschreitbarkeit“ (ebd.) besteht. Letztere beruht auf dem elterlichen Verbot, sich in den Tannengrund zu begeben, das von Marie gebrochen wird. Die Gegend jenseits des Flusses bildet dagegen keinen Aktions-, sondern einzig einen Erinnerungsraum oder, genauer gesagt, einen ,erinnerten Raum‘. Das ehemalige Zuhause von Martin und Brigitte, das ausschließlich im ersten Elterngespräch und im letzten Satz des Textes auftaucht, fungiert hier nur als trüber, fruchtloser Gegensatz zum jetzigen Lebensort der Hauptfiguren - dem heiteren, gutsituierten Dorf diesseits des Flusses. Folgendes Schema soll diese dreidimensionale Raumteilung summarisch veranschaulichen:

\begin{tabular}{|l|l|l|}
\hline \multicolumn{2}{|c|}{ Diesseits des Flusses } & \multicolumn{1}{|c|}{ Jenseits des Flusses } \\
\hline Schöne, fruchtbare Gegend, & $\begin{array}{l}\text { Der geheimnisvolle Tannen- } \\
\text { grund - die einzige „Schat- }\end{array}$ & $\begin{array}{l}\text { Trauriger und dürrer Ort, an } \\
\text { dem Maries Eltern früher ge- }\end{array}$ \\
wo Martin und Brigitte zusam- \\
men mit ihrer Tochter Marie \\
ein sorgloses, glückliches Le- \\
ben führen.
\end{tabular}


Die Tatsache, dass der finstere, furchterregende Tannengrund sich ausgerechnet diesseits des Flusses befindet und den ansonsten einwandfreien, vorbildlichen Raum durch das bloße Dasein-Faktum befleckt, ist strukturell wie semantisch relevant, denn eben dieses depressiv wirkende Außenseitergebiet entpuppt sich später als wunderschönes Elfenreich. Letzteres bildet damit eine Art Binnenraum, welcher von der tüchtigen, christlichen Bauernwelt sorgfältig abgegrenzt ist.

Die Grenzen markieren «Strom» und «Hügel mit Birnbaum». Im Zentrum liegt, von einem Bach eingeschlossen und von einem Hund bewacht, das Elfenreich. Ein Tannenwald und ein mit Wächtern besetzter Wald schirmen es kreisförmig von der Außenwelt ab. Als Reich der Phantasie grenzt es sich zwar nach außen ab, nach innen entfaltet es aber eine unendliche Tiefe, in der auch die Chronologie der Alltagszeit aufgehoben ist. Sieben Jahre verkürzen sich hier auf etwas mehr als eine Nacht, in der allerdings nicht geschlafen, sondern, wie es ausdrücklich heißt, bis zum Morgen «gelustwandelt» [...] wird. (Kremer, in: Stockinger; Scherer 2001: 511-512)

Die Bezeichnung „Reich der Phantasie“ ist hier allerdings nur teilweise akzeptabel. Als ungebundene Willkür bzw. kindliche Freiheit des schöpferischen Geistes ist die Phantasie zweifelsohne ein bedeutendes Element der Elfenwelt. Sobald man aber Maries zwischenräumlichen Grenzübergang in psychoanalytischen Kategorien zu behandeln beginnt, spricht man Tiecks Text das Märchenhafte ab, so dass Die Elfen zur puren Allegorie einer gestörten Adoleszenz reduziert werden. Die Erfahrungen der Protagonistin im Elfenreich bilden keineswegs „kulturell legitimierte Ausdrucksformen für unbewusste Vorgänge" (Würzbach, in: Nünning 2004: 60). Vielmehr sind sie als Metapher einer romantisch-mystischen Offenbarung zu verstehen. Albert Béguin sieht die Gründe dafür, dass ausgerechnet Marie dazu erwählt wurde, in ihrer kindischen Unschuld (vgl. Béguin 2011: 280). Dies stimmt allerdings nur einerseits. Zurecht stellt andererseits Judit Kováts fest, dass es sich im Fall von Elfen um keine naive, unreflektierte Kindergeschichte handelt, sondern um ein „formal und gedanklich“ komplexes, vielschichtiges Werk (Kováts: 450, Zugriff am 22.06.2017). Doch gleich nach dieser völlig zutreffenden Bemerkung scheint die Forscherin in ein anderes Extrem zu fallen, indem sie das im Text Erzählte übermäßig psychologisiert und in den Termini eines ,post-freudschen` Trauma-Berichts interpretiert:

Im Grunde geht es in der Märchennovelle Die Elfen um die Geschichte einer Mutter und ihrer Tochter, deren trauriges Schicksal mit ihrem lebensfernen Charakter zusammenzuhängen scheint, und deren Seelenzustand als eine Krankheitsdiagnose mit psychologischer Genauigkeit beschrieben wird. Das Wunderbare wird in der Geschichte auf das knappe Handlungsgerüst projiziert in der Form der Vision. Das Kind Marie erlebt die Zeit bei den Zigeunern als einen Aufenthalt in der Elfenwelt. Der wüste Garten des Zigeunervolkes verwandelt sich im Bewusstsein des Mädchens in einen bunten paradiesischen und wird mit allen möglichen Requisiten einer Märchenwelt ausgestattet [...]. (ebd. 455)

Der Text ,rebelliert' jedoch gegen eine derartige Interpretationsmöglichkeit ganz deutlich, und zwar nicht nur im Schlussteil der Erzählung, in dem auch Maries Ehemann Andres ,zu seinem Erstaunen“ (Tieck 1983: 27) Zeuge der El- 
fenexistenz wird, sondern noch wesentlich früher, gleich nach Maries Rückkehr in die Menschenwelt. Als Marie nämlich von ihren Eltern und anderen erwachsenen Dorfbewohnern nach ihrem Aufenthalt während der sieben Jahre befragt wird, betont der (keineswegs unzuverlässige) Erzähler den erzwungenen Charakter dieser Gespräche:

Man fragte von neuem, man drang in sie, doch die, des Verbotes eingedenk, konnte keine Antwort geben. Man legte ihr fast die Erzählung in den Mund, daß sie sich verirrt habe, auf einen vorbeifahrenden Wagen genommen und an einen fremden, fernen Ort gebracht sei, wo sie den Leuten den Wohnsitz ihrer Eltern nicht habe bezeichnen können; wie man sie nachher nach einer weit entlegenen Stadt gebracht habe, wo gute Menschen sie erzogen und geliebt; wie diese nun gestorben, und sie sich endlich wieder auf ihre Geburtsgegend besonnen, eine Gelegenheit ergriffen habe und so zurückgekehrt sei. (ebd. 21)

Es scheint also, dass Kováts sich bei der psychoanalytischen Elfen-Deutung genau dieser ,In-den-Mund-legen-Methode` bedient, die im Text so ausführlich beschrieben (und latent kritisiert) wird. Dabei ist zu betonen, dass, während die in den Elfen dargestellte Ereignisversion wenigstens realistisch klingt, so ist Kováts’ Annahme, Marie habe die sieben Jahre „bei den Zigeunern“ verbracht (siehe das Zitat oben), rein logisch gesehen, eher abwegig. Man kann sich schließlich schwer vorstellen, dass ein verlaufenes Kind sieben Jahre lang hinter einem Tannenwald lebt, ohne entdeckt zu werden, obwohl sich dieser Tannenwald in der Nähe vom Heimatdorf des Kindes befindet. Maries Geschichte mag ,keine Familiengeschichte, sondern die eines emanzipierten Kindes“ (Kreuzer 1983: 119) sein; eine Krankheitsgeschichte ist sie aber keinesfalls. Die „Unvereinbarkeit des Erzählten mit unserem Wirklichkeitsverständnis“ (Vogt 2006: 19) sollte somit als ein vom Autor bewusst eingesetzter und für die gesamte (früh)romantische Literatur durchaus kennzeichnender narratologischer Kunstgriff interpretiert werden.

Das Reich, in dem das abenteuerlustige und ungehorsame Mädchen ganz unerwartet ihre magische Zuflucht findet, ist in erster Linie ein anderer Raum. Die „Konfrontation mit dem Fremden“(Würzbach 2006: 65) bedeutet damit zugleich eine „Auseinandersetzung mit Normen und Werten“ (ebd.), die mit den Gesetzen der Menschenwelt wenig zu tun haben. Nicht zufällig wird bereits am Anfang der Novelle die ehemalige Gegend jenseits des Flusses erwähnt, welche Maries Eltern früher bewohnt haben. Der gravierende, von Maries Mutter explizit ausgedrückte Unterschied zwischen dem alten und dem neuen Lebensort bereitet den Leser auf einen noch stärkeren, qualitativ-essentiellen Unterschied zwischen der Menschenund der Elfenwelt vor. Ein sehr wichtiger Aspekt der Elfen-Komposition besteht darin, dass sich neben der „Zweigeteiltheit des Wirklichen“ (Kováts: 454, Zugriff am 22.06.2017) auch ein dritter, übernatürlicher Raum als existent erweist: der glühend-blühende Elfenraum hinter der dunklen Tannenwaldfassade. Diese Fassade sorgt ihrerseits für weitere interpretatorische Missverständnisse. So scheint folgende Behauptung von Kováts auf den ersten Blick treffend zu sein: 
Im Werk besteht eine Analogie zwischen dem vegetativen und dem humanen Dasein, bzw. eine Wechselwirkung zwischen der Seele des Menschen und den Kräften der Natur. Der Dichter selbst ist der Überzeugung, dass „Bäume und Blumen eine Physiognomie besitzen, die wir wahrzunehmen vermögen“. Der Bach fließt „schwermütig“ vorüber, die ländliche Umgebung ist „,heiter“, der „,abgelegene Fleck hinter den Tannenbäumen ist „traurig“ [...]. (ebd. 456)

So sehr sich die Postulierung der Übereinstimmung von Mensch und Natur für die Analyse eines romantischen Kunstmärchens auch eignet, ja für selbstverständlich gehalten werden kann, ist gerade diese „Analogie“ in Tiecks Elfen trügerisch, denn die menschlichen Augen (wenigstens die eines erwachsenen Menschen) sehen hier die Welt falsch oder - anders formuliert - sie sehen nicht die ganze Welt. Die Menschen können den inneren Raum hinter dem äußeren nicht erblicken. Wie Christoph Brecht richtig bemerkt: „Im leeren Raum, den die literarisch geordnete Welt zum Zentrum hat, hat das kombinatorische Spiel des Märchens seinen Ort; hier werden die Zeichen für das Wirkliche vertauscht und geraten in eine eigene, vernünftig nicht mehr kontrollierbare Bewegung " (Brecht 1993: 121). Der „schwermütige“ Bach drückt daher keine emotionale Harmonie von der Menschen- und Naturwelt aus, sondern zeugt im Gegenteil von einer blinden Enge anthropozentrischer Wirklichkeitsbetrachtung. Es sind ja einzig die Dorfmenschen (mit Ausnahme von Marie), die behaupten, der trübe Tannengrund werde vom „Zigeunervolk“ (Tieck 1983: 8) bewohnt, welchem man eher intuitiv als berechtigt kein Vertrauen schenkt. Maries Mutter Brigitte drückt dieses distanzierte bis feindliche Verhältnis, diese unbewusste ,Elfenphobie‘ sehr prägnant aus:

[...] man kann ihnen doch eben nichts Böses nachsagen, nur ist es bedenklich, daß sie sich nicht zur Kirche halten und man auch eigentlich nicht weiß, wovon sie leben, denn der kleine Garten, der noch dazu ganz wüst zu liegen scheint, kann sie unmöglich erhalten, und Felder haben sie nicht. (ebd.)

In der Tat ist also weder der Bach „schwermütig“ noch der Tannengrund ein trauriger „abgelegener Fleck“, genauso wie dessen Bewohner keine Zigeuner, sondern magische Wesen - die Elfen - sind. Indem man, Tiecks Text interpretierend, dem menschlichen Sichtpunkt objektives Betrachtungsvermögen verleiht, verfehlt man die Kernaussage des Märchens. Um diese zu bestimmten, sollte man sich dem zweiten Teil der Geschichte, d.h. Maries Leben in der Menschenwelt nach dem Aufenthalt im Elfenreich näher zuwenden.

\section{Regeln und Gesetze - Die verstörte Anonymität des Wunderbaren}

Wenn Judit Kováts sagt: „Marie kehrt in die wirkliche Welt mit dem Bewusstsein eines achtjährigen Kindes zurück“ (Kováts: 461, Zugriff am 22.06.2017), so wird dabei ein bedeutsamer Faktor übersehen, nämlich dass dieses achtjährige Kind 
die Zeit seiner Abwesenheit in einer anderen, ,parallelen Wirklichkeit' verbracht hat. Man könnte natürlich meinen, Marie habe dadurch die Adoleszenz-Phase sozusagen ,übersprungen', so dass sie sich nach ihrer Rückkehr nicht etwa wie ein Teenager-Mädchen, sondern eher wie ein kleines Kind verhält. Der Text gibt jedoch wiederum keine Anlässe für eine solche Interpretation. Im Gegenteil heißt es bei Tieck:

Oft dachte sie mit inniger Sehnsucht an ihren Aufenthalt hinter den Tannenbäumen zurück; sie blieb still und ernst. So schön auch alles war, was sie umgab, so kannte sie doch etwas noch Schöneres, wodurch eine leise Trauer ihr Wesen zu einer sanften Schwermut stimmte. Schmerzhaft traf es sie, wenn der Vater oder ihr Mann von den Zigeunern und Schelmen sprachen, die im finstern Grunde wohnten; oft wollte sie sie verteidigen, die sie als Wohltäter der Gegend kannte [...] aber sie zwang das Wort jedesmal in ihre Brust zurück. So lebte sie das Jahr, und im folgenden ward sie durch eine junge Tochter erfreut, welche sie Elfriede nannte, indem sie dabei an den Namen der Elfen dachte. (Tieck 1983: 23)

So wird in den Elfen die Gestalt der sechzehnjährigen Marie skizziert. Man sieht daran deutlich, dass die Zeit im Elfenreich keine emotionale Rückständigkeit bzw. geistige Unterentwicklung ausgelöst hat. Verglichen mit den Gleichaltrigen, zeigt sich die ,stille und ernste“ Protagonistin viel reifer als sie, obschon ihre frühere heiter-sorglose Lebenseinstellung durch „das zwiespältige Weltempfinden“ (Stölzel 2013: 38), das schon für die Hauptfiguren von Der blonde Eckbert charakteristisch war, ersetzt wird. Es wäre demnach logisch zu vermuten, dass ihre Adoleszenz nicht ausfällt, sondern sich in einer Welt bzw. Dimension vollzieht, deren Struktur und Existenz völlig anderen Regeln unterliegen als die Welt der Menschen (der Verzicht auf die Bezeichnung ,reale Welt" ist hier durchaus intentional, denn als Märchen schließt Die Elfen jegliche Teilung erzählter Räumlichkeit in ,real“" und ,irreal“" aus). Was sind es aber für Regeln?

Nach Ingrid Kreuzer ist das elfische Paradies ,,von statischer Langeweile erfüllt" (Kreuzer 1983: 120). Einerseits lässt sich diese Ansicht anhand des Textes leicht beweisen, denn die Bewohner des Elfenreiches verbringen ihre Zeit (die hier übrigens als Synonym der Freizeit fungiert) tatsächlich nur mit Spielen und Lustwandeln. Andererseits sollte man dabei beachten, dass der Erzähler die Geschehnisse zwar möglichst objektiv, aber auch distanziert darstellt. Im Unterschied zur Protagonistin hat er keinen Kontakt zur magischen Wirklichkeit (anders als z.B. der Erzähler in E.T.A. Hoffmanns Der goldne Topf). Wir sehen die Welt der Elfen nicht einmal mit Maries Augen, denn um diesen Effekt zu erlangen, sollte man zur homodiegetischen Ich-Erzählform oder wenigstens zur personalen Erzählperspektive greifen. Bei Tieck verhält sich die Erzählinstanz eher neutral. Maries Erlebnisse und Erfahrungen werden dem Leser so berichtet, als wenn der Erzählende ein unbeteiligter Dritter wäre, der ein Gespräch mit Marie geführt und die wichtigsten Informationen aufgeschrieben hat. Wenn wir also der Elfenwelt Statik und Langeweile zuschreiben, so resultiert diese Bewertung aus unserer Außenperspektive, die ihrerseits durch die Außenperspektive des Erzählers bedingt ist. 
Denn Marie scheint sich im Elfenreich keineswegs zu langeweilen, steht es doch im Text: ,„Ich möchte lachen, und mir graut“, sagte Marie“ (Tieck 1983: 17) oder noch ausdrücklicher: ,[...] ich will hier bleiben [...], denn hier ist es schön, auch finde ich hier das beste Spielzeug und dazu Erdbeeren und Kirschen, draußen ist es nicht so herrlich“" (ebd.).

Kehren wir nun zurück zu der Frage nach den Regeln und Gesetzen, die in der elfischen Welt herrschen. Es heißt bei Kováts: „Als Marie ihrem Mann von den sieben Jahren ihrer Abwesenheit erzählt, beginnt der Zerfall ihrer Identität. Auf der Ebene des Wunderbaren heißt es: als sie ihrem Mann vom Geheimnis ihrer Kindheit [...] erzählt, verletzt sie das Gesetz der Natur, und zieht die Strafe der Elfen, die eine höhere moralische Instanz verkörpern, auf sich.“ (Kováts: 467, Zugriff am 11.07.2017). Dieses „Gesetz der Natur“ ist eine bemerkenswerte, zum üblichen romantischen Diskurs durchaus passende - und doch etwas diffuse Formulierung. Die Gleichsetzung von Elfenreich und Naturwelt gilt nämlich nur unter bestimmten Einschränkungen. Einerseits scheint das paradiesische, arbeitsfreie Leben von Elfen im Vergleich zum tüchtigen, von Leistungen und Erwartungen dominierten Menschenraum wesentlich lockerer und zutraulicher. Andererseits ist diese wunderbare Wirklichkeit keineswegs offen zugänglich, so dass nur Auserwählte dorthin gelangen können, und zwar nur für eine - nach Elfenmaßstäben rechnend - kurze Zeit. Noch signifikanter ist dabei das Schweigegebot, welches Marie nach ihrer Rückkehr ins Heimatdorf streng halten soll. Aus irgendeinem unaussprechlichen (oder wenigstens unausgesprochenen) Grund dürfen die Menschen nichts über die Elfenwelt erfahren. „Worüber man nicht sprechen kann, davon soll man schweigen oder singen" (Safranski 2015: 102), wie es Rüdiger Safranski in seinem Romantik-Monographie in Bezug auf Der blonde Eckbert so schön pointiert. Auch sollten die Dorfbewohner nicht wissen, woher die fröhliche Fruchtbarkeit diesseits des Flusses kommt und wem sie ihr Wohlleben eigentlich verdanken. Die Situation, in der die Elfen von Menschen als gefährliche Zuwanderer, als gott- und ehrenlose Zigeuner betrachtet werden, ist - aus der Perspektive der Elfen selbst - paradoxerweise nicht nur akzeptabel, sondern gar erwünscht: Eine solche Ordnung der Dinge bildet ja, wie es sich letztendlich herausstellt, die Grundbedingung für die ungestörte Koexistenz zweier Wirklichkeiten. „Wer an das Geheimnis rührt, beschwört das Unheil herauf" (Gebhardt 1997: 153), um es mit Arnim Gebhardt zu formulieren.

Der Elfenraum ist demnach zu kompliziert konstruiert und konzipiert, um ihn als bloße Metapher des Naturraums zu betrachten. Man kann sogar nicht eindeutig feststellen, dass diese Welt den Kindern offensteht, für die Erwachsenen dagegen geschlossen bleibt, denn nicht alle Kinder finden den Weg ins magische Elfenreich. Der gehorsame, vorsichtige, rational eingestellte Andres wäre z.B. kein potentieller Kandidat für derlei zwischenweltliche Kontakte. Es scheint, nebenbei bemerkt, nicht ohne Bedeutung zu sein, zu welchen Jahreszeiten Maries erzwungene Verlobung und Heirat mit Andres stattfindet: „Im Herbste gab Marie 
den dringenden Bitten des Andres nach: sie ward seine Braut und im Winter mit ihm verheiratet“ (Tieck 1983: 22). In der Elfenwelt herrscht hingegen „ein ewiger Sommer und Frühling“" (ebd. 17).

\section{Mann vs. Frau, Diesseits vs. Jenseits - Schlussfolgerung}

Interessanterweise wird das Wunderbare in Ludwig Tiecks Die Elfen ausschließlich den Frauenfiguren zuteil. Die männlichen Gestalten haben, rein semantisch gesehen, eine zweitrangige Bedeutung, obwohl ihr Auftreten für den Aufbau des Textes durchaus relevant ist: „[...] Martin dient als Strukturfigur: Er intoniert den Textanfang und überlebt das Textende, wenn er am Textende aus der dargestellten Gegend abwandert. Er fungiert so als umgrenzende Randfigur des «Wirklichkeits» bereichs, dem die elfische Mitte einliegt [...]“ (Kreuzer 1983: 119), so Ingrid Kreuzer. Es ist aber nicht nur Martin, der das Textende überlebt, sondern auch sein Schwiegersohn Andres, wohingegen alle wichtigsten Frauenfiguren - Brigitte, Marie und Elfriede - sterben. In diesem Kontext sind die letzten drei Sätze des Märchens beachtenswert: „Marie stand oft auf dem Platze vor der Hütte und beweinte das entschwundene Glück. Sie verzehrte sich, wie ihr Kind, und folgte ihm in einigen Jahren. Der alte Martin zog mit seinem Schwiegersohne nach der Gegend, in der er vormals gelebt hatte“" (Tieck 1983: 30). Die Geschichte endet somit mit der Erwähnung jenes Raumes, welcher auch am Anfang angesprochen wurde. Einen logischen, handlungsbedingten Grund, warum die männlichen Figuren in die dürre, düstere Gegend jenseits des Flusses zurückkehren, scheint es kaum zu geben, denn nach dem Weggang der Elfen gibt es ja keinen Unterschied mehr zwischen beiden Räumen. Man könnte jedoch annehmen, dass dadurch die den ganzen Text durchströmende Dichotomie von Dies- und Jenseits auf eine höhere, metaphysische Stufe erhoben wird. Während Martin und Andres sich kreisartig zwischen den einst konträren und nun völlig identischen Räumen dies- und jenseits des Flusses bewegen, befreien sich Marie und Elfriede von der dualistischen Beengtheit der erzählten Welt und begeben sich in jene Wirklichkeit, deren Benennung sogar für einen romantischen Dichter ein allzu kühner Versuch wäre, geschweige denn für einen Literaturwissenschaftler.

\section{Literatur}

Béguin, Albert (2011): Dusza romantyczna i marzenie senne. Gdańsk.

Borais, Alexander (2011): Karel Čapeks Poetik der noetischen Detektion im Kontext seiner Pragmatismus-Rezeption. Hamburg.

Brecht, Christoph (1993): Die gefährliche Rede. Sprachreflexion und Erzählstruktur in der Prosa Ludwig Tiecks. Tübingen. 
Gebhardt, Armin (1997): Ludwig Tieck. Leben und Gesamtwerk des „,Königs der Romantik“. Marburg.

Hölter, Achim (2005): Über Weichen geschickt und im Kreis gejagt. In: Kremer, Detlef (Hrsg.): Die Prosa Ludwig Tiecks. Bielefeld. S. 69-91.

Kováts, Judit: Grenzübergänge des Wirklichen in Ludwig Tiecks Märchen „Die Elfen“. http://epa. oszk.hu/02100/02137/00021/pdf/EPA02137_ISSN_1219-543X_tomus_15_fas_3_2010_ ger_447-468.pdf (Zugriff am 20.06.2017, 22.06.2017, 11.07.2017).

Kremer, Detlef (2005): Einsamkeit und Schrecken. Psychosemiotische Aspekte von Tiecks „Phantasus "-Märchen. In: Kremer, Detlef (Hrsg.): Die Prosa Ludwig Tiecks. Bielefeld. S. 53-69.

Nünning, Vera / Nünning, Ansgar (Hrsg.) (2004): Erzähltextanalyse und Gender Studies. Stuttgart/ Weimar.

Meißner, Thomas (2007): Erinnerte Romantik. Ludwig Tiecks „Phantasus“. Würzburg 2007.

Kreuzer, Ingrid (1983): Märchenform und individuelle Geschichte. Zu Text- und Handlungsstrukturen in Werken Ludwig Tiecks zwischen 1790 und 1810. Göttingen.

Lotman, Jurij (1993): Die Struktur künstlerischer Texte. Stuttgart.

Lüer, Edwin (1997): Aurum und Aurora: Ludwig Tiecks „Der Runenberg” und Jakob Böhme. Heidelberg.

Safranski, Rüdiger (2009): Romantik. 6. Auflage. Frankfurt am Main.

Schirmunski, Wiktor (1996): Немецикии романтизм и современная мистика (Deutsche Romantik und moderne Mystik). Sankt Petersburg.

Stockinger, Claudia; Scherer, Stefan (Hrsg.) (2011): Ludwig Tieck. Leben Werk-Dichtung. Berlin/ Boston.

Stölzel, Simone (2013): Nachtmeerfahrten. Die dunkle Seite der Romantik. Berlin.

Tieck, Ludwig (1983): Der Runenberg. Märchen und Novellen. Rudolstadt.

Tieck, Ludwig (1985): Werke in zwei Bänden. Zweiter Band. Berlin/Weimar.

Vogt, Jochen (2006): Aspekte erzählender Prosa. 9. Auflage. München.

Würzbach, Natascha (2004): Raumdarstellung. In: Nünning, Vera / Nünning, Ansgar (Hrsg.): Erzähltextanalyse und Gender Studies. Stuttgart/Weimar.

Würzbach, Natascha (2006): Raumerfahrung in der klassischen Moderne. Großstadt, Reisen, Wahrnehmungssinnlichkeit und Geschlecht in englischen Erzähltexten. Trier.

Germanica Wratislaviensia 143, 2018

(C) for this edition by CNS 